\title{
Lacrimal canaliculitis due to Arachnia (Actinomyces) propionica
}

\author{
D. V. SEAL, ${ }^{1} \mathrm{~J}_{\text {MCGILL }}{ }^{2}$ D. FLANAGAN, ${ }^{2}$ AND B. PURRIER ${ }^{1}$ \\ From the ${ }^{1}$ Public Health Laboratory, Southampton, and ${ }^{2}$ Southampton Eye Hospital
}

SUMMARY The clinical and microbiological findings in a chronic case of lacrimal canaliculitis due to Arachnia propionica are described. Bacterial culture and identification should be performed in the investigation of the disease in order to establish the role of $A$. propionica and other specific actinomycetes at the acute as well as at the chronic stage.

Mycotic canaliculitis has been recognised for many years as an uncommon clinical entity which usually responds to surgery and iodine. 146 cases have been reported from 1854 to 1972, in which a single canaliculus has usually been involved, the lower in 105 cases and the upper in $41 .^{1}$ The disease has been considered in the past as due to streptothrix, leptothrix, or nocardia organisms but is now thought to be due to anaerobic actinomycetes, though the species involved has not often been identified. ${ }^{23}$

Actinomyces propionicus was first isolated from a case of lacrimal canaliculitis in 1960, when it was characterised and described as a new species. ${ }^{4}$ It has since been identified from another 5 cases, ${ }^{5-7}$ but the course of the disease in relationship to the microbiological findings was not reviewed. The organism was reclassified as Arachnia propionicus ${ }^{8}$ but has now been designated Arachnia propionica.

We report the course of a patient with a 4-year history of recurrent purulent discharge from the left upper punctum, from whom an anaerobic actinomycete has been isolated repeatedly. It has defied eradication by surgery and antibiotics, though we have now obtained a remission of the patient's symptoms.

\section{Case report}

CLINICAL SUMMARY

In 1975 a 14-year-old boy presented with purulent conjunctivitis of the left eye that had failed to respond to topical chloramphenicol. There was swelling and induration round the left upper canaliculus and punctum, with blocking of the nasolacri-

Correspondence to Dr D. V. Seal, Public Health Laboratory, Level B, South Laboratory Block, Southampton General Hospital, Tremona Road, Southampton SO9 4XY. mal duct. A culture of the discharge grew Haemophilus influenzae, sensitive to gentamicin, while actinomycetes could be neither seen on examination of pus nor cultured from it. The patient was started on topical gentamicin and Polyfax (polymyxin B and bacitracin).

The swelling and discharge persisted. After 2 months the left upper punctum and canaliculus were explored under general anaesthesia. White cheesy material was removed from the canaliculus, leaving a cavity $2 \times 2 \mathrm{~mm}$ wide. Histological examination showed chronic granulomatous tissue and bacilli. Microscopy of pus swabs showed numerous polymorphonuclear cells and Gram-positive branching bacilli (actinomycetes). No aerobic bacteria were cultured, but an anaerobic actinomycete was grown, which was found to be sensitive to penicillin and chloramphenicol. At that time it was not fully identified. The patient was started on oral penicillin V $500 \mathrm{mg} 4$ times a day for 2 weeks and topical penicillin 100000 units per $\mathrm{ml} 4$ times a day to the left eye.

The patient was continued on topical penicillin for 3 months, by which time the infection had completely subsided. The swelling round the left upper canaliculus had resolved and the nasolacrimal duct was again patent. The topical penicillin was discontinued. After a further 3 months the patient was considered to be free of infection and was discharged from the clinic.

The patient presented again 17 months later complaining of a yellow discharge of 1 month's duration from the medial corner of the left eye. On examination there was purulent discharge from the left upper punctum. This was cultured and Haemophilus influenzae was grown, sensitive to ampicillin and chloramphenicol. The patient was started on 
topical penicillin 100000 units per $\mathrm{ml} 4$ times a day to the left eye, together with topical chloramphenicol 3-hourly. After 1 month he was considered to have improved with only minimal discharge from the left punctum. The topical antibiotic therapy was discontinued.

The patient again presented after 14 months, complaining of intermittent discharge in the left eye. On examination there was a nontender swelling round the left upper canaliculus. A purulent discharge could be expressed from the left upper punctum, which contained many polymorphonuclear cells together with actinomycetes, appearing as scanty Gram-positive diphtheroidal and filamentous branching rods (Fig. 1). Aerobic culture yielded a scanty growth of Staphylococcus aureus, resistant to penicillin but sensitive to cloxacillin, chloramphenicol, and gentamicin. Anaerobic culture yielded a growth of an actinomycete sensitive to penicillin, cephalosporin, cloxacillin, chloramphenicol, erythromycin, fucidin, rifampicin, and tetracycline but resistant to gentamicin, neomycin, soframycin, sulphonamide and trimethoprim. The patient was started on topical penicillin 100000 units per $\mathrm{ml}$ 4 times a day together with topical gentamicin. The actinomycete was subsequently identified as Arachnia propionica. After 1 month there was some improvement, with the discharge diminishing in frequency and amount; topical antibiotics were continued.

However, after 4 months an intermittent discharge

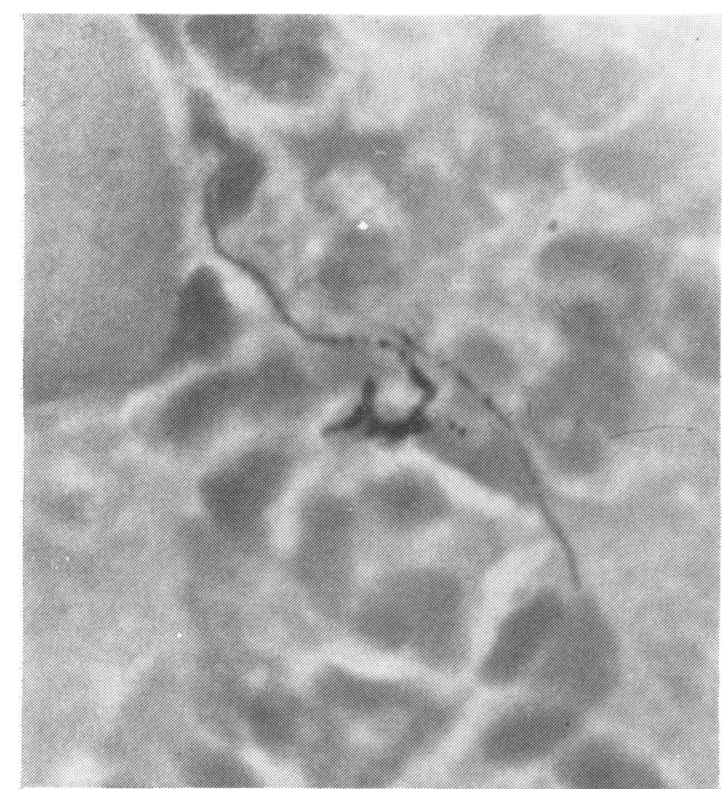

Fig. 1 Gram stain of actinomycetes and polymorphonuclear cells. had recurred that again yielded a growth of Arachnia propionica. The patient was started on oral penicillin V $500 \mathrm{mg} 4$ times a day. After a further month $A$. propionica was still isolated but had remained sensitive to penicillin and cloxacillin. The patient was therefore changed to oral flucloxacillin, $500 \mathrm{mg}$ 4 times a day, which would have greatly increased the tissue level of antibiotic. However, this failed to stop the discharge and regular penicillin syringing was begun. After 2 months culture of a wash-out of the left sac failed to grow $A$. propionica or other bacteria. With further penicillin syringing the discharge has ceased, with a remission in the clinical condition.

\section{MICROBIOLOGICAL EXAMINATION}

Conjunctival and pus swabs were examined by Gram stain and cultured for aerobic and anaerobic bacteria. Culture for aerobic bacteria was performed by plating the swabs on to blood and chocolate agars, which were incubated in $5 \%$ carbon dioxide at $37^{\circ} \mathrm{C}$ for 48 hours, and by placing the swab in glucose broth, which was subcultured after 24 hours. Antibiotic sensitivity tests were carried out using a control organism by Stokes's technique. ${ }^{9}$

Culture for anaerobic bacteria was performed by plating the swabs on to blood and chocolate agars, which were incubated anaerobically for 5 days at $37^{\circ} \mathrm{C}$. Baird and Tatlock anaerobic jars were used with palladiumised aluminium crystals as a catalyst and a gas mixture of $10 \%$ carbon dioxide and $90 \%$ hydrogen. Antibiotic sensitivity tests were carried out by disc diffusion on blood agar under anaerobic conditions.

Under anaerobic conditions small dry white 'spider' colonies, $1 \mathrm{~mm}$ in size, appeared on the surface of the agar after 5 days. On microscopy cells appeared as Gram-positive diphtheroidal and filamentous branching rods (Fig. 2). This typical appearance was noted by Brock et al. ${ }^{6}$ On subsequent subculture growth of this actinomycete was extremely slow on solid media, but it was found to grow over a 2-week period in thioglycollate broth. Typical 'bread crumb' colonies were formed in this medium. The actinomycete grew best anaerobically, but scanty growth was obtained aerobically with $5 \%$ carbon dioxide after 2 weeks' incubation. This actinomycete is thus a microaerophile rather than a strict anaerobe.

The actinomycete was characterised as follows: facultative aerobic Gram-positive branching rod; negative catalase, urea, gelatin, and aesculin tests; weakly positive indole test; fermentation of glucose, lactose, maltose, mannose, mannitol, raffinose, and sucrose; nonfermentation of arabinose, cellobiose, glycerol, melizitose, rhamnose, salicin, and xylose; 


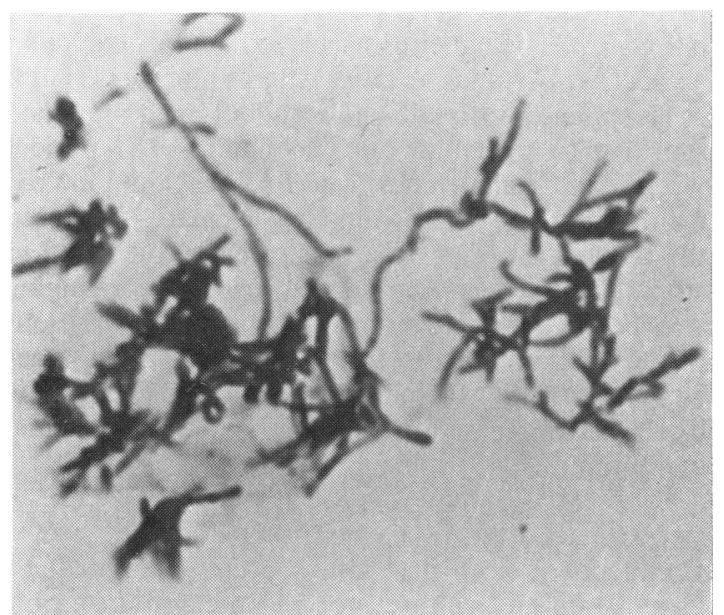

Fig. 2 Gram stain of actinomycetes from laboratory culture.

fermentation products of glucose were found to be acetic, propionic, and small quantities of lactic acid.

These results are sufficient to identify the isolate as Arachnia propionica from detailed tables. ${ }^{458}$ A. propionica differs from Actinomyces spp. with its production of large quantities of propionic acid in broth culture and the presence of diaminopimelic acid in its cell wall; it also has different surface antigens. ${ }^{6} A$. propionica has similar morphology to Actinomyces spp. including Actinomyces israelii, which it also resembles on culture. However, it differs by failing to ferment arabinose, cellobiose, salicin, and xylose. Both positive and negative results are quoted for the indole test, ${ }^{458}$ which may be explained by our 'weakly positive' result.

\section{Discussion}

Pine et al. ${ }^{10}$ have isolated Propionibacterium acnes or anaerobic diphtheroids from the lacrimal duct of 29 out of 55 normal and diseased eyes, without canaliculitis, showing that there is an anaerobic environment within the duct. However, they failed to isolate anaerobic actinomycetes from these eyes, though they did isolate Actinomyces israelii from 1 case of canaliculitis and suggested that it was in a parasitic rather than invasive stage. Anaerobic actinomycetes, including Arachnia propionica, are normal flora of the mouth, particularly the teeth, and may reach the lacrimal canaliculus indirectly from saliva via the fingers or directly via the nasal passages and nasolacrimal duct.

Ruys, ${ }^{11}$ Elliot, ${ }^{2}$ Ridley and Smith, ${ }^{12}$ GibsonMoore, ${ }^{13}$ and Smith $^{14}$ have described canaliculitis in 28 patients. They cultured purulent discharge expressed from the punctum, or concretions removed at surgery from the lacrimal canaliculus, with aerobic and anaerobic techniques. However, except in the case of 1 patient in which Actinomyces bovis was suggested they identified only the morphology of the bacteria isolated under various conditions, as was the custom at that time. The descriptions indicate that the bacteria isolated have been anaerobic actinomycetes, though 2 authors referred to them as leptothrix or streptothrix organisms. Pine et al. $^{\mathbf{3}}$ identified such actinomycetes from 2 patients as being Actinomyces israelii, though after further investigation they reclassified 1 isolate as Arachnia (Actinomyces) propionica. Aerobic and anaerobic culture, with further identification of anaerobic actinomycetes thus isolated, is required in the investigation of the disease. Characteristics of actinomycetes should be compared with those given in tables by Gerencser and Slack. ${ }^{5}$ Antibiotic sensitivity tests should be performed. Results can be compared with those given by Garrod ${ }^{15}$ for Actinomyces israelii and by Lerner ${ }^{16}$ for the pathogenic actinomycetes. We agree with Brock et al. ${ }^{6}$ that it is logical to retain the name 'actinomycosis' for chronic suppurative disease caused by both Actinomyces spp. and Arachnia spp.

The details of the 6 other cases of Arachnia propionica canaliculitis are as follows.

Pine et al. ${ }^{3}$ investigated a 14-year-old boy with acute conjunctivitis of the left eye that had been discharging for the previous 1 year. He was treated with gentamicin drops, when the acute conjunctivitis responded but the discharge persisted. The superior canaliculus was explored twice and many particles were removed from a $2 \times 3 \mathrm{~mm}$ cavity, which grew Arachnia propionica. The patient was given tetracycline drops for 5 weeks with no evidence of recurrence.

Gerencser and Slack ${ }^{5}$ investigated a 61-year-old woman with a 3-year history of exudation at the inner canthus of the left eye, with repeated episodes of swelling and acute infection. The lacrimal system was probed and irrigated repeatedly but without relief. Exudate containing concretions was cultured and yielded Arachnia propionica.

Brock et $a .^{6}$ (with Dr L. Pine) investigated a patient treated with sulphonamide drops for 6 months for discharge from the left eye. Curettage of the left upper canaliculus and removal of concretions, which grew Arachnia propionica, was performed on several occasions. Tetracycline drops were given, and the patient recovered.

Brock et al. ${ }^{6}$ (with Dr C. T. Dolan) investigated a 55-year-old man with a 4-year history of an infected tear duct. Arachnia propionica was cultured from purulent material from the canaliculus. 
Brock et al. ${ }^{6}$ (with Dr M. A. Gordon) isolated Arachnia propionica from a patient with lacrimal canaliculitis, but no details were given.

Jones and Robinson ${ }^{7}$ isolated Arachnia propionica together with Staphylococcus aureus from a canalicular culture of a patient with subacute dacryocystitis, but no details were given.

Isolation of Arachnia propionica from the canaliculus thus appears to be associated with chronic infection that may be difficult to eradicate. However, identification of actinomycetes is at present attempted only from patients who do not respond to therapy, and it may be that this actinomycete can also cause canaliculitis that does respond, without recurrence, to simple surgical excision. This needs to be established by identifying isolates cultured from acute cases. In chronic cases surgical removal of the concretions with application of iodine, a bactericidal antiseptic, to the site is recommended. Systemic treatment with penicillin or erythromycin should be given together with topical treatment of antibiotic irrigations. Tetracycline drops have apparently been found successful. Antibiotic drops that should not be used are gentamicin, neomycin, soframycin, and sulphonamide, since this and other anaerobic actinomycetes are resistant to them.

We wish to thank Mr R. Nash, Department of Oral Microbiology, London Hospital Medical College, for carrying out fermentation and gas chromatographic tests on our behalf on this bacterium.

We also thank Miss P. J. Stoffell for typing the manuscript.

\section{References}

1 Francois J, Rysselaere M. Oculomycoses. Springfield: Thomas, 1972.

2 Elliot AJ. Streptothricosis of the lachrymal canaliculi. Am J Ophthalmol 1941; 24: 682-6.

3 Pine L, Hardin H, Turner L, Roberts SS. Actinomycotic lachrymal canaliculitis. Am J Ophthalmol 1960; 49: 127888.

4 Buchanan BB, Pine L. Characterization of a propionic acid producing actinomycete, Actinomyces propionicus, sp. nov. J Gen Microbiol 1962; 28: 305-23.

5 Gerencser MA, Slack JM. Isolation and characterization of Actinomyces propionicus. J Bacteriol 1967; 94: 109-15.

6 Brock DW, Georg LK, Brown JM, Hicklin MD. Actinomycosis caused by Arachnia propionica. Am J Clin Pathol 1973; 59: 66-77.

7 Jones DB, Robinson NM. Anaerobic ocular infections. Trans Am Acad Ophthalmol Otolaryngoi 1977; 83: 309-31.

8 Pine L, Georg LK. Reclassification of Actinomyces propionicus. Int J Systemat Bacteriol 1969; 19: 267-72.

9 Stokes EJ, Waterworth PM. Antibiotic sensitivity tests by diffusion methods. ACP Broadsheet 1972; No. 55.

10 Pine L, Shearin WA, Gonzales CA. Mycotic flora of the lachrymal duct. Am J Ophthalmol 1961; 52: 619-25.

11 Ruys A. Charlotte. Concretions in a lachrymal canaliculus caused by Actinomyces. Br J Ophthalmol 1935; 19: 385-9.

12 Ridley F, Smith C. Leptotrichosis conjunctivae. $\mathrm{Br} \mathrm{J}$ Ophthalmol 1952; 36: 328-9.

13 Gibson-Moore J. Actinomycosis of the canaliculi with invasion of tissue in one case. Br J Ophthalmol 1952; 36: 522-4.

14 Smith CH. Ocular actinomycosis. Proc $R$ Soc Med 1953; 46: 209-12.

15 Garrod LP. The sensitivity of Actinomyces israeli to antibiotics. $\mathrm{Br} \mathrm{Med} J$ 1952; i: 1263-4.

16 Lerner PI. Susceptibility of pathogenic actinomycetes to antimicrobial compounds. Antimicrob Agents Chemother 1974; 5: 302-9. 